combinations having the largest effect sizes (OR range 2.32-3.17) relative to the low force/low repetition exposure combinations.

Conclusions Use of job-title based exposures was useful for demonstrating associations with prevalent CTS. Jobs with combined high exposures of force and repetition showed consistently greater risk of CTS compared to jobs with lower exposure levels.

\section{BIAS IN EXPOSURE ASSESSMENT FROM WORST-CASE SELECTION OF WORKPLACES IN OSHA'S INTEGRATED MANAGEMENT INFORMATION SYSTEM DATABANK IMIS}

'Philippe Sarazin, ${ }^{2}$ Laurel Kincl, ${ }^{3}$ Igor Burstyn, ${ }^{1,4}$ Jérôme Lavoué. 'University of Montreal, Montreal, Quebec, Canada; ${ }^{2}$ Oregon State University, Corvallis, Oregon, USA; ${ }^{3}$ Drexel University, Philadelphia, Pennsylvania, USA; ${ }^{4}$ University of Montreal Hospital Research Centre, Montreal, Quebec, Canada

\subsection{6/oemed-2014-102362.151}

Objectives The Integrated Management Information System (IMIS), with over $1 \mathrm{M}$ measurements taken by inspectors of the Occupational Safety and Health Administration, is an important source of information for occupational epidemiology. We assessed the association of the reason for conducting inspection with the reported levels of chemical exposure in IMIS.

Method Time weighted averaged measurements made during each of the un-programmed inspection types (employee complaint, referral by safety officer, follow-up, monitoring) were compared to those made during programmed inspections for 50 chemicals. Ratios of the median of detected results (dM), and the differences in the proportion of non-detects $(\mathrm{dPr})$ for each category compared to the programmed inspections were calculated for each chemical.

Results The analysis included 218916 measurement records. $32 \%$ were collected during programmed inspections, $48 \%$ - complaints, $13 \%$ - referral, $5 \%$ - follow-up, and 2\% - monitoring. The detected concentrations were similar for complaint $(\mathrm{dM}=0.98$, interquartile range across chemicals, $\mathrm{IQR}=$ $[0.83 ; 1.11])$ and referral $(\mathrm{dM}=0.91, \mathrm{IQR}=[0.76 ; 1.08])$ inspections and greater for follow-up $(\mathrm{dM}=2.18, \mathrm{IQR}=[1.38 ; 3.13])$ and monitoring $(\mathrm{dM}=1.59, \mathrm{IQR}=[1.24 ; 2.44])$ inspections relative to presumed representative inspections. Similarly, the proportion of non-detects were similar to programmed inspections during complaint-driven $(\mathrm{dPr}=3 \%, \mathrm{IQR}=[-1 ; 8])$ and referrals $(\mathrm{dPr}=0 \%$, $\mathrm{IQR}=[-6 ; 5])$ and lower during follow-up $(\mathrm{dPr}=-11 \%, \mathrm{IQR}=[-$ $19 ;-2])$ and monitoring $(\mathrm{dPr}=-8 \%, \mathrm{IQR}=[-12 ; 3])$ inspections.

Conclusions Despite the absence of consistent differences across chemicals for the most frequent categories, exposure levels during non-programmed surveys can be significantly higher than those obtained during presumably representative measurement campaigns. Great care has to be taken in determining typical exposure distributions from OSHA's IMIS data.

\section{EXPOSURE REGISTRIES AS A TOOL FOR EPIDEMIOLOGY}

${ }^{1}$ Victoria H Arrandale, ${ }^{2}$ Stephen Bornstein, ${ }^{1,3}$ Paul A Demers. 'Occupational Cancer Research Centre, Toronto, ON, Canada; ${ }^{2}$ Memorial University, St. John's, NL, Canada; ${ }^{3}$ University of Toronto, Toronto, ON, Canada

\subsection{6/oemed-2014-102362.152}

Objectives In Canada, there is growing interest in the use of registries for hazard and disease surveillance because they provide an opportunity for primary prevention. Registry data can also be a valuable tool for epidemiology. Here we report the strengths and limitations of using exposure registry data for epidemiology.

Method Eight exposure registries were reviewed: five from Canada, two from the USA and one from Finland. They were compared based on overall goals, exposure information, registration, recruitment, and health information collected. The potential use of registry data in epidemiology was evaluated. Key considerations for designing a registry that facilitates secondary data use were identified.

Results The eight registries varied significantly. Data from four had been previously used in epidemiology. In three cases exposure measurements were available within the registry; in one, health information was also collected. Registries that have mandatory registration are more likely to contain sufficient data for use in epidemiological studies in contrast to voluntary registries that may fail to capture a large or representative portion of the exposed population. In order to permit later linkage of registry data with health information, consent must be obtained in advance and privacy legislation must be taken into consideration.

Conclusions Most exposure registries are not designed with secondary data uses in mind and, as a result, the use of exposure registry data in epidemiological studies can be problematic. Given the large investment involved in launching a new registry, opportunities to leverage the data for epidemiological purposes should be explored in the planning stages.

\section{SPONTANEOUS ABORTION IN FLIGHT ATTENDANTS}

${ }^{1}$ Barbara Grajewski, 'Elizabeth Whelan, 'Christina Lawson, 'Misty Hein, 'Martha Waters, 1 Jeri Anderson, ${ }^{1}$ Leslie MacDonald, ${ }^{2}$ Christopher Mertens, ${ }^{1}$ Chih-Yu Tseng, ${ }^{1}$ Cassinelli II Rick, 'Lian Luo. ${ }^{1}$ National Institute for Occupational Safety and Health, US Centers for Disease Control and Prevention, Cincinnati, OH, USA; ${ }^{2}$ National Aeronautics and Space Administration, Hampton, VA, USA

\subsection{6/oemed-2014-102362.153}

Objectives Flight attendant occupational exposures include cosmic ionising radiation and circadian disruption. We wanted to determine whether these and other occupational exposures were associated with spontaneous abortion among female flight attendants.

Method Female flight attendants from three US airlines in three cities were interviewed. Company records of over 1.9 million individual flights during the study period were assessed for exposure to galactic cosmic radiation, solar particle event radiation, and circadian disruption. Measures of physical job demands and other occupational factors were obtained from the interview. Cox proportional hazards regression models were adjusted for age, parity, and nonflying status.

Results Among 2273 women interviewed, 840 pregnancies among 673 women met inclusion criteria. There was evidence to suggest that cosmic radiation exposure of $0.1 \mathrm{mGy}$ or more may be associated with increased risk of spontaneous abortion in weeks $9-13$ of the first trimester (odds ratio $(\mathrm{OR})=1.74 ; 95 \%$ confidence interval (CI) $0.95-3.20$ ). The risk of a first trimester spontaneous abortion was significantly increased with $15 \mathrm{~h}$ or more of flying during home base normal sleep hours $(\mathrm{OR}=1.54$; $95 \%$ CI 1.07-2.21) and with high physical job demands $(\mathrm{OR}=2.49 ; 95 \%$ CI 1.49-4.16).

Conclusions Spontaneous abortion was associated with several flight attendant occupational exposures. This is the first report of these associations based on a quantitative assessment of distinct exposures generated from individual flight records. 